\title{
Effect of family function on adolescent social stress response
}

Binggen Zhu; Zhiqi Sun; Xiaodan Ren; Xiandi Pan, Xudong Zhao. Pudong New Area Mental Health center, Tongji University School of Medicine, Shanghai, China

Adolescents who lived in the bad family environment were more likely to have misadjusted physiological stress response system and mental health problems, such as anxiety, depression, and impulsion etc. Whether "intact" families with poor function affect the adolescent stress responses hasn't been studied intensively yet. We tested 95 (16-19 years; male:41, female:54) first-grade technical secondary school students by Family Assessment Device (FAD) to find out their family functions, and by Center for Epidemiological Studies Depression Scale (CES-D), StateTrait Anxiety Inventory (STAI), Aggression Questionnaire (AQ) and Adolescent Life Event Scale (ALES) to evaluate their mental health and life events. Then we used modified Trier Social Stress Test (TSST) and detected salivary cortisol contents by the ELISA.

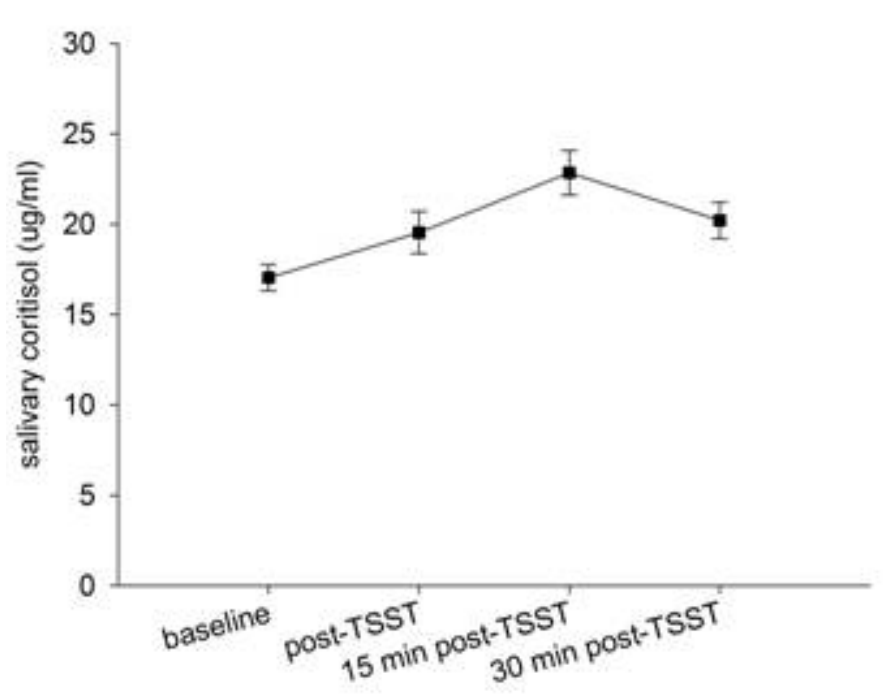

Fig. 1 The salivary cortisol levels of four time points [15 minutes before TSST (TSST1), 0 minutes (TSST2), 15 minutes (TSST3) and 30 minutes (TSST4) after TSST] in the modified TSST.

We found: 1 . There was a significant salivary cortisol difference among four time points of the TSST(Fig.1), implying that the modified TSST was established. 2. Salivary cortisol of TSST1, TSST2 and TSST4 had a significant correlation with communication dimension of family function, and salivary cortisol of TSST2 had a significant correlation with affective reaction (Table 1). 3.TSST1 \& TSST2's salivary cortisol level in the poor family communication group was significantly higher than that of normal group, and TSST2's salivary cortisol level in the poor family affective reaction group was significantly higher than that of normal group (Fig.2 A\&B). In contrast, TSST3's salivary cortisol level in the poor problem solving group was obviously lower than that of normal group, although it has not reached a significant level (Fig.2 C).
4. Stress situations reflected by the TSST had no significant correlation with sex, age, mental health problems (e.g depression, anxiety and aggression) of students, and socioeconomic indexes of parents, such as marital status, economic status, educational background, employment.
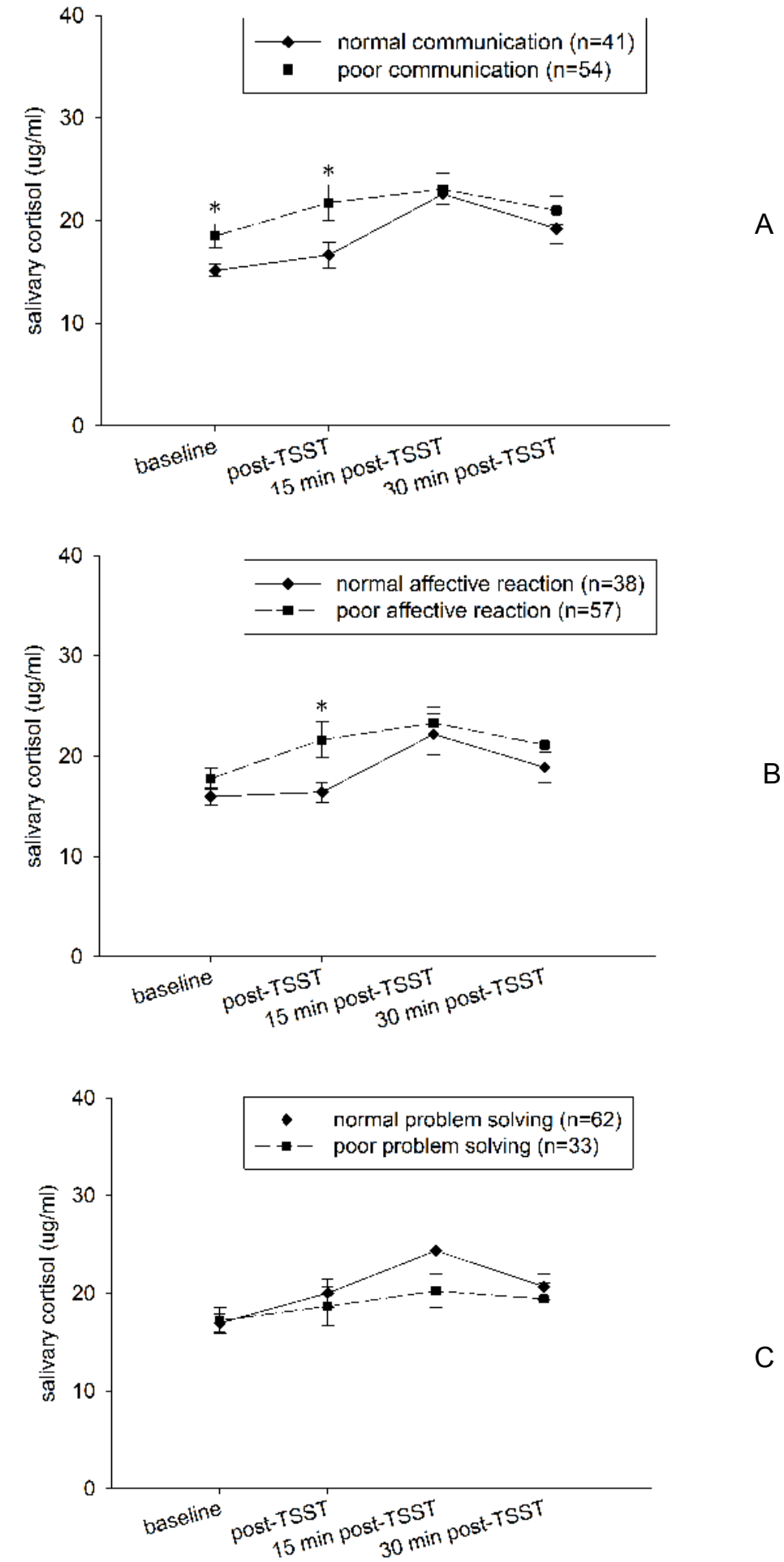

Fig.2 The effect of dimensions of family function [communication (A), affective reaction $(B)$ and problem solving $(C)$ ] on salivary cortisol levels. ${ }^{*} \mathrm{P}<0.05$

These results implied that adolescents with poor family communication were in a higher basic state of stress, adolescents with poor family affective reaction had stronger stress response, while adolescents with poor family problem solving probably had lower stress response. Therefore, the family function and its associated dimensions might exert various influences on stress response in adolescents.

Acknowledgements: the Health and Family Commission of Pudong New Area, Shanghai, China (PWZzk2017-20)

Table 1 correlation analysis between salivary cortisol levels of four time points in the modified TSST and family functions

\begin{tabular}{|c|c|c|c|c|c|c|c|c|}
\hline Time points & \multicolumn{2}{|c|}{ TSST1 } & \multicolumn{2}{|c|}{ TSST2 } & \multicolumn{2}{|c|}{ TSST3 } & \multicolumn{2}{|c|}{ TSST4 } \\
\hline Correlation analysis & Pearson correlation & Sig & Pearson correlation & Sig & Pearson correlation & Sig & Pearson correlation & Sig \\
\hline Problem solving & 0.041 & 0.691 & -0.036 & 0.727 & -0.153 & 0.144 & 0.000 & 0.999 \\
\hline communication & $0.231^{*}$ & 0.025 & $0.323^{\star \star \star}$ & 0.001 & 0.073 & 0.489 & $0.229^{*}$ & 0.027 \\
\hline role & 0.175 & 0.090 & 0.072 & 0.488 & -0.132 & 0.207 & 0.007 & 0.950 \\
\hline Affective reaction & 0.172 & 0.095 & $0.261^{*}$ & 0.011 & 0.021 & 0.840 & 0.124 & 0.233 \\
\hline Affective intervention & 0.177 & 0.086 & 0.083 & 0.425 & -0.095 & 0.367 & -0.033 & 0.754 \\
\hline Behavior control & 0.080 & 0.442 & 0.033 & 0.750 & -0.081 & 0.439 & 0.015 & 0.888 \\
\hline General function & 0.064 & 0.536 & -0.048 & 0.646 & -0.162 & 0.120 & -0.084 & 0.422 \\
\hline
\end{tabular}

${ }^{* * *} \mathrm{P}<0.001,{ }^{* *} \mathrm{P}<0.01,{ }^{*} \mathrm{P}<0.05$ 